\title{
Research on Adaptive Neural Network Control System Based on Nonlinear U-Model with Time-Varying Delay
}

\author{
Fengxia Xu, Yao Cheng, Hongliang Ren, and Shili Wang \\ School of Computer and Control Engineering, Qiqihar University, Qiqihar 161006, China \\ Correspondence should be addressed to Fengxia Xu; xufengxia_hit@163.com
}

Received 6 June 2014; Accepted 31 July 2014; Published 13 August 2014

Academic Editor: Ligang Wu

Copyright ( 2014 Fengxia Xu et al. This is an open access article distributed under the Creative Commons Attribution License, which permits unrestricted use, distribution, and reproduction in any medium, provided the original work is properly cited.

\begin{abstract}
U-model can approximate a large class of smooth nonlinear time-varying delay system to any accuracy by using time-varying delay parameters polynomial. This paper proposes a new approach, namely, U-model approach, to solving the problems of analysis and synthesis for nonlinear systems. Based on the idea of discrete-time U-model with time-varying delay, the identification algorithm of adaptive neural network is given for the nonlinear model. Then, the controller is designed by using the Newton-Raphson formula and the stability analysis is given for the closed-loop nonlinear systems. Finally, illustrative examples are given to show the validity and applicability of the obtained results.
\end{abstract}

\section{Introduction}

Since most physical systems and processes in the real world are nonlinear, researchers have been devoting their efforts to seeking an effective means of controlling nonlinear systems. Among the many developments, there are growing interests in the adaptive control of complex nonlinear systems. On the other hand, in control systems, time-varying delay is almost the inherent feature of general physical systems, which often degrades the system's performance and even causes instability. Therefore, time-varying delays have received great attention in recent years, and many researchers have studied various analytical techniques and developed many synthesis methods for time-delay systems. For instance, stability analysis is carried out in [1-7] and the controllers are designed in [8-15]. The time-varying delay being widespread in nonlinear control system, it is often difficult to establish accurate mathematical model because of its complex dynamic characteristics, so it is difficult to design a practical controller. For the nonlinear time-varying delay systems, it is a hot and difficult problem to design a controller with high control precision and good system stability in control theory. The difficulty in modeling the nonlinearities makes nonlinear time-varying delay systems much more difficult to control than linear systems, so a general high accuracy model becomes critical.

The main difficulty in nonlinear time-varying delay control system design lies in the lack of general control oriented modelling framework, and the model is very important in the design of nonlinear control system; at the same time, the model must be able to describe various time-varying delay systems sufficiently. Nonlinear U-model represents a large class of smooth nonlinear system by using the way of time-varying delay parameters polynomial and the nonlinear plants without any loss of original nonlinear characteristics, so the nonlinear time-varying delay system is represented by U-model. U-model constructs a general model for the controller design in the traditional nonlinear system and builds a bridge between the design method of the nonlinear dynamic systems and the linear control systems. In methodology, U-model provides a straightforward routine to design nonlinear control systems by using linear control system design techniques.

Because the identification process of the time-varying delays system is often required online, it is difficult to solve the identification problem by using traditional methods, and because the artificial neural network has strong ability of 
identification of nonlinear multivariable systems, it is widely used in system identification. Zou and Wang [16] proposed an identification algorithm based on recurrent neural network, which selected NARMAX model to describe discrete nonlinear time-varying system, the parameters of NARMAX model were identified by regression neural network online. Pillonetto [17] presented a method of time-varying autoregressive system identification in the reproducing kernel Hilbert space. Hsiao [18] proposed an algorithm to identify time-varying systems by using maximum a posteriori estimation. Liu et al. [19] initiated a novel approach for simultaneously identifying the topological structure and unknown parameters of uncertain general complex networks with time delay. For the identification of time-varying delay systems, the precision of identification is not only related to identification algorithm, but also has direct relationship with the choice of system model, considering the U-model can be well represented for a class of discrete time-varying delay nonlinear systems, and has the characteristics of general and high precision, which, combined with artificial neural network, has strong ability of nonlinear multivariable system identification; this paper presents a method to identify the time-varying delays parameters of $\mathrm{U}$-mode by neural network.

In the study of nonlinear controller design, Zhu and Guo [20] proposed a pole assignment controller design scheme for a class of nonlinear controlled plant with known structure and parameters, which used Diophantine equations corresponding polynomial to complete pole assignment based on U-model. Shafiq and Butt [21] proposed an internal model control method based on U-model, which is used to solve a class of known dynamic nonlinear plants, considering that the parameters are known, so U-model can be applied directly. Khan and Shafiq [22] put forward an adaptive inverse method based on the minimum variance (LMS) of U-model and applied to control the complex nonlinear industrial process system. The structural parameters of the nonlinear plant are unknown. Wu et al. [23] presented an adaptive stochastic nonlinear system control based on U-model, applying weighted iterative least square method to identify the unknown plant, by using control of pole assignment. Motivated by these earlier developments, in this paper, we propose a new approach for the control design of nonlinear time-varying delay systems with unknown structure and use neural network to identify the time-varying delay parameters of U-model. The adaptive controller is designed by NewtonRaphson formula. According to the identification results of neural network, the parameters of the Newton Raphson formula are adjusted online.

The remaining portion of the paper is organized as follows. Section 2 introduces U-model representation of nonlinear systems. The design of nonlinear time-varying delay control system is described in Section 3. Based on the results obtained in Section 3, illustrative examples are given in Section 4 to demonstrate the effectiveness of the theoretical results. Finally, some concluding remarks are given in Section 5 .

\section{U-Model Representation of Nonlinear Systems}

In Zhu and Guo [20], U-model was firstly proposed to approximate a class of nonlinear time-varying delay model, which can be represented as

$$
y(k)=\sum_{j=0}^{m} \alpha_{j}\left(k-d_{j}\right) u^{j}(k-1)+e(k),
$$

where $m$ is the degree of model input $u(k-1)$ and $\alpha_{j}\left(k-d_{j}\right)$ is a function of past inputs $u\left(k-d_{1}\right), \ldots, u\left(k-d_{j}\right)$ and outputs $y(k-1), \ldots, y(k-n), d_{j}$ is the delay parameter of $\alpha_{j}\left(k-d_{j}\right)$, and $e(k), \ldots, e(k-n)$ are errors. Adjusting the polynomial, it can be concluded that the control model with time-varying delay parameter can be expressed as the input power series.

From formula (1), it can be seen that the U-model has simple mathematical form; to apply linear control system design methodologies to the nonlinear model, a further transformation is applied as follows:

$$
\begin{gathered}
U(k)=y(k), \\
U(k)=\sum_{j=0}^{m} \alpha_{j}\left(k-d_{j}\right) u^{j}(k-1)+e(k) .
\end{gathered}
$$

Formula (3) is a mathematical expression of U-model.

In order to estimate time-varying delay parameter $\Theta(k)$ of the U-model, $\mathrm{U}$-model vector representation is given as

$$
y(k)=U(k)=\Theta(k) \Psi(k)+e(k),
$$

where $\Theta(k)=\left[\alpha_{0}\left(k-d_{0}\right), \ldots, \alpha_{j}\left(k-d_{j}\right)\right]$ is the delay parameter and $\Psi(k)=\left[1, u(k-1), \ldots, u^{j}(k-1)\right]^{T}$.

$\mathrm{U}$-model can be a good representation of a class of nonlinear time-varying delay plant. The transformation of U-model will not lose any characteristics of the original nonlinear model, compared with the other control model in $\mathrm{Xu}$ et al. [24]; representation of the U-model is more practical, and controller design methodologies derived from linear systems can be developed accordingly to design nonlinear discrete time systems.

\section{Design of Nonlinear Time-Varying Delay Control System}

3.1. Identification of Neural Network. Because neural network has the advantages of approximating any nonlinear system, it is often used for the identification of nonlinear systems. The time-varying delay parameters $\Theta(k)=\left[\alpha_{0}\left(k-d_{0}\right), \ldots, \alpha_{j}(k-\right.$ $\left.d_{j}\right)$ ] can be regarded as the weights of the neural network, through online adjustment of weights making the identification error meet the system requirements of precision, thus, obtaining time-varying delay parameters of the system; the time-varying delay parameters will be used as the input of the controller, and adjusting the output of the controller online, the structure of nonlinear neural network identification is shown in Figure 1. 


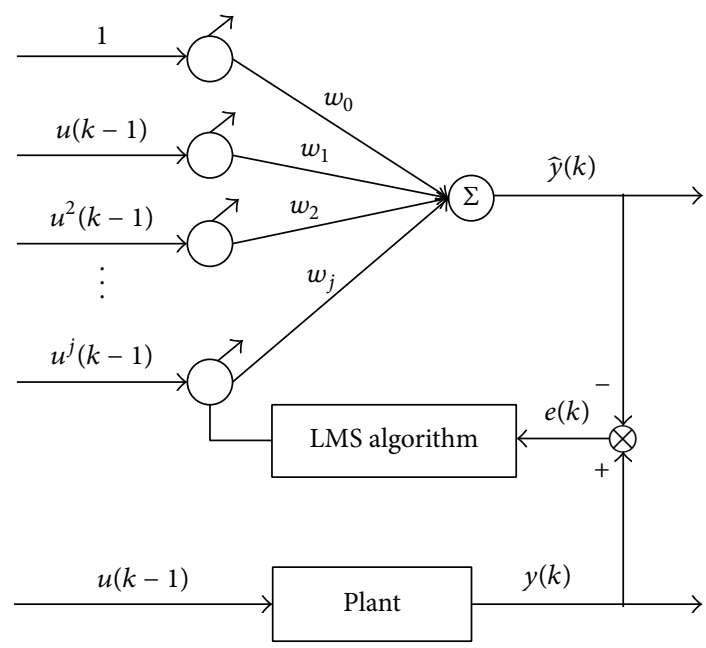

FIGURE 1: The structure of nonlinear neural network identification.

Widrow-Hoff learning rule (LMS algorithm) is used to adjust the weights of the neural network identification. Input vector $\Psi(k)=\left[1, u(k-1), \ldots, u^{j}(k-1)\right]^{T}$, the weights vector $\Theta(k)=\left[\alpha_{0}\left(k-d_{0}\right), \ldots, \alpha_{j}\left(k-d_{j}\right)\right]$, and the transfer function of the output node uses the linear function; the output of the neural network is

$$
y(k)=U(k)=\Theta(k) \Psi(k)+e(k),
$$

where the weights vector $\Theta(k)=W(k)=\left[w_{0}(k)\right.$, $\left.w_{1}(k), \ldots, w_{j}(k)\right]$. In addition, in the process of identification, the error $e(k)$ can be identified as a part of the parameter $\alpha_{0}\left(k-d_{0}\right)$. In the process of weights adjustment, error signal is

$$
e(k)=y(k)-W(k) \Psi(k) .
$$

The objective function is defined as

$$
E(w)=\frac{1}{2} e^{2}(k) .
$$

According to formula (7), finding the partial derivative of $E(w)$, we obtain

$$
\frac{\partial E}{\partial w}=-\Psi(k) e(k)
$$

Therefore, according to the gradient descent method, the adjustment rules of the weights are as follows:

$$
w(n+1)=w(n)+\eta \Psi(k) e(k),
$$

where $\eta$ is the learning rate.

In the identification of neural network, it has a great influence on convergence speed of the algorithm to select suitable learning rate and accuracy of the error, if the learning rate selected is too small, after a finite times adjustment, the weights may not reach the control precision required but reduce convergence speed of the algorithm. If the error precision selected is too small, it will lead to reduce the speed of convergence. For fast time-varying delay system, it has a great impact on the stability and control precision of the controller to select appropriate learning rate and accuracy of the error. In addition, when the highest order of $u(k-1)$ is higher than the recognition structure's, we can identify it as a part of $\alpha_{0}\left(k-d_{0}\right)$.

3.2. The Design of Adaptive Control System. After sampling of nonlinear time varying delay plant, it can be represented as $\mathrm{U}$-model structure of discrete form, this paper uses neural network to identify the time-varying delay parameters of $U$ model. The controller adjusts the output according to the parameters obtained by the neural network identification. The adaptive controller is designed by adopting the NewtonRaphson formula and adjusts the parameters according to the results of identification online. The output of adaptive controller and the input of controlled plant are reciprocal processes in the system, when the controller and the controlled plant match exactly; namely, the output of the system always can track the input accurately. The structure diagram of adaptive control system is as shown in Figure 2: where $U(k)$ is the input of the system, $u(k-1)$ is the output of the controller, $y(k)$ is the output of the system, $\hat{y}(k)$ is the output of the neural network identification, and $e(k)$ is the system error.

The Newton-Raphson formula is used to solve the roots of equation in Zhu et al. [25]; in this paper, the Newton-Raphson formula is used to design controller to make the design of controller simplify, it is the most widely used method to solve the nonlinear equations; compared with other methods, this method has high convergence speed. General mathematical description of the Newton-Raphson formula is as follows:

$$
x_{k+1}=x_{k}-\frac{f\left(x_{k}\right)}{f^{\prime}\left(x_{k}\right)}
$$

where $f\left(x_{k}\right)$ is a nonlinear function, and the NewtonRaphson formula can be used to solve nonlinear equation (3) to obtain the root; the equation is described as follows:

$$
\begin{aligned}
u_{k+1}(k-1) & \\
& =u_{k}(k-1)-\frac{\phi\left[u_{k}(k-1)\right]-U(k)}{d \phi\left[u_{k}(k-1)\right] / d u(k-1)} \\
& =u_{k}(k-1)-\frac{\sum_{j=0}^{M} \alpha_{j}(k-j) u_{k}^{j}(k-1)-U(k)}{d\left[\sum_{j=0}^{M} \alpha_{j}(k-j) u_{k}^{j}(k-1)\right] / d u(k-1)},
\end{aligned}
$$

where the subscript $k$ is the times of iterative algorithms. In the Newton-Raphson formula, in order to ensure the real roots of nonlinear equations, we need to define the conditions in the algorithm: avoiding the denominator is zero and the iterative algorithm has faster convergence speed.

3.3. Stability Analysis. The stability is the basic requirement in the design of control system; in order to check the validity of the adaptive neural network control method proposed, 


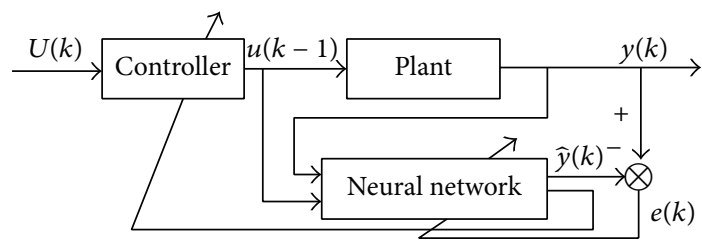

FIGURE 2: Adaptive control system structure diagram.

suppose the nonlinear time-varying delay plants are stable. The controller is designed by using the Newton-Raphson formula; in order to ensure that the Newton-Raphson formula always has a real root, the convergence condition needs to be limited.

If the U-model expression is such as $y(k)=\alpha_{0}\left(k-d_{0}\right)+$ $\alpha_{1}\left(k-d_{1}\right) u(k-1)$, the equation always has a real root; if the $\mathrm{U}$-model expression is such as $y(k)=\alpha_{0}\left(k-d_{0}\right)+\alpha_{1}(k-$ $\left.d_{1}\right) u(k-1)+\alpha_{2}\left(k-d_{2}\right) u^{2}(k-1)$ and when the equation has no real root, in order to keep the equation having a real solution, make $\alpha_{0}\left(k-d_{0}\right)=U(k)$; ensure the equation always has a real root: $u(k-1)=-\alpha_{2}\left(k-d_{2}\right) / \alpha_{1}\left(k-d_{1}\right)$.

For the high order equation $y(k)=\alpha_{0}\left(k-d_{0}\right)+\alpha_{1}(k-$ $\left.d_{1}\right) u(k-1)+\cdots+\alpha_{j}\left(k-d_{j}\right) u^{j}(k-1)$, it can always ensure that the equation has a real root by limiting the condition of solution of the equation. As long as there is a real root, namely, the controller is stable, the whole control system designed is stable.

\section{The Simulation Research}

There are four simulation examples of adaptive neural network control system based on time-varying delay nonlinear U-model; in the simulation examples, the parameters of neural network identification are given as follows: the maximum times of weights iteration is 1000 , the learning rate is 0.01 , the error precision is 0.0001 , and the weight change is 0.0001 . We choose initial weights $\Theta(k)=\left[\alpha_{0}\left(k-d_{0}\right) \alpha_{1}\left(k-d_{1}\right)\right]=\left[\begin{array}{ll}1 & 1\end{array}\right]$ in model 1 and $\Theta(k)=\left[\alpha_{0}\left(k-d_{0}\right) \alpha_{1}\left(k-d_{1}\right) \alpha_{2}\left(k-d_{2}\right)\right]=$ $\left[\begin{array}{lll}1 & 2 & 1\end{array}\right]$ in model 2. The parameters of the controller are given as follows: the maximum times of iterations is 100 , the minimum change of iterations is 0.001 ; when the change of the iteration is less than the minimum change, stop the iterative algorithm, the final value for adaptive controller will be obtained.

Model 1. Choosing nonlinear time-varying delay system: $y(k)=\alpha_{0}\left(k-d_{0}\right)+\alpha_{1}\left(k-d_{1}\right) u(k-1)$, where $\alpha_{0}\left(k-d_{0}\right)=$ $0.2 y(k-1) u(k-2) ; d_{0}=2 ; \alpha_{1}\left(k-d_{1}\right)=0.8, d_{1}=0$. The nonlinear model of the plant is obtained: $y(k)=0.2 y(k-$ $1) u(k-2)+0.8 u(k-1)$; the system contains only the zero order and one order of $u(k-1)$ can be regarded as a linear system. After choosing sine and square wave signal as the input signal, the tracking curve diagram of the system is as shown in Figures 3 and 4.

Model 2. Add two orders of $u(k-1)$ to the nonlinear timevarying delay system in model 1 ; the expression of nonlinear

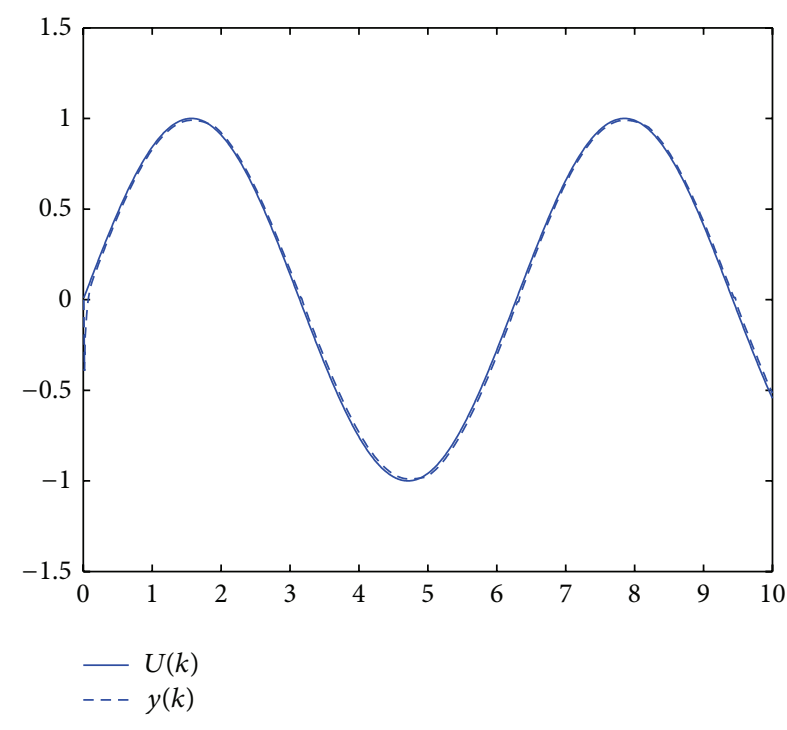

FIGURE 3: The response curve of sine signal.

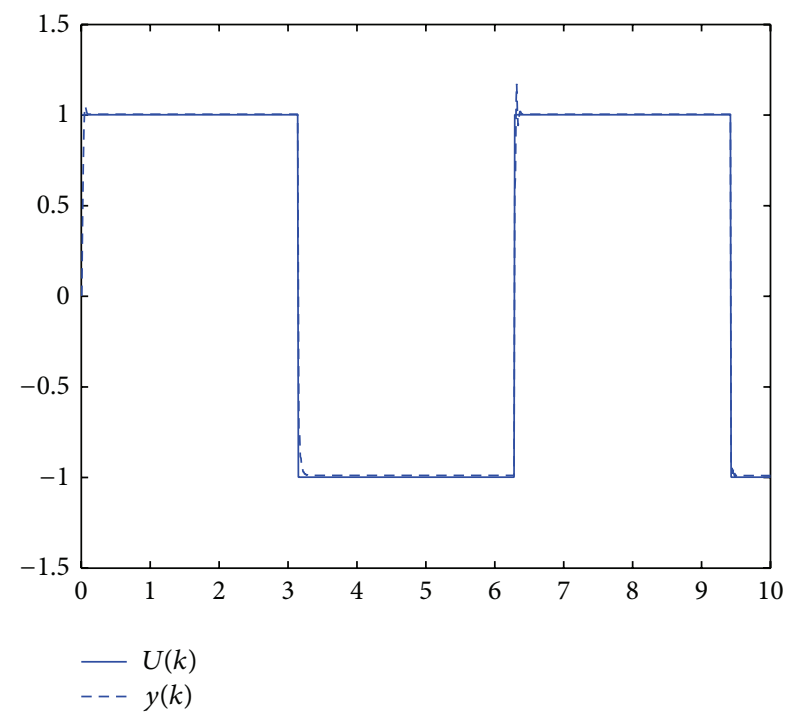

Figure 4: The response curve of square wave signal.

time-varying delay system is $y(k)=0.2 y(k-1) u(k-2)+$ $0.8 u(k-1)+0.02 u^{2}(k-2)$; it contains a nonlinear term. After choosing sine and square wave signal as the input signal, the tracking curve diagram of the system is as shown in Figures 5 and 6.

Model 3. Choosing nonlinear time-varying delay system: $y(k)=\alpha_{0}\left(k-d_{0}\right)+\alpha_{1}\left(k-d_{1}\right) u(k-1)+\alpha_{2}\left(k-d_{2}\right) u^{2}(k-1)$, there are two delay coefficients in this system, where $\alpha_{0}\left(k-d_{0}\right)=$ $0.1 y(k-1) u\left(k-d_{0}\right), d_{0}=2 ; \alpha_{1}\left(k-d_{1}\right)=0.8 u(k-2), d_{1}=2$; $\alpha_{2}\left(k-d_{2}\right)=-0.3 y(k-1), d_{2}=0$. The nonlinear model of the plant is obtained: $y(k)=0.1 y(k-1) u(k-2)+0.8 u(k-2) u(k-$ $1)-0.3 y(k-1) u^{2}(k-1)$. After choosing sine and square wave 


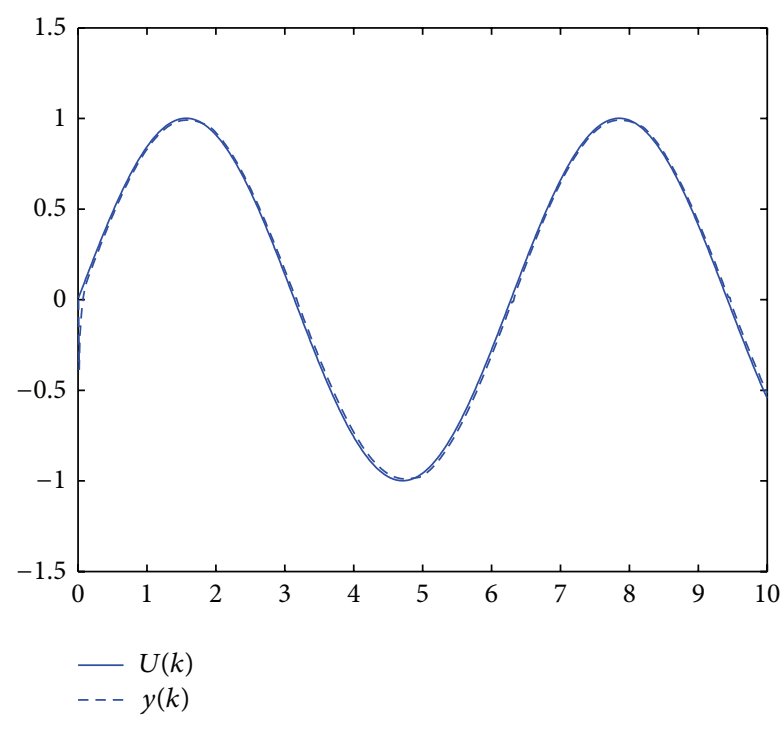

FIGURE 5: The response curve of sine signal.

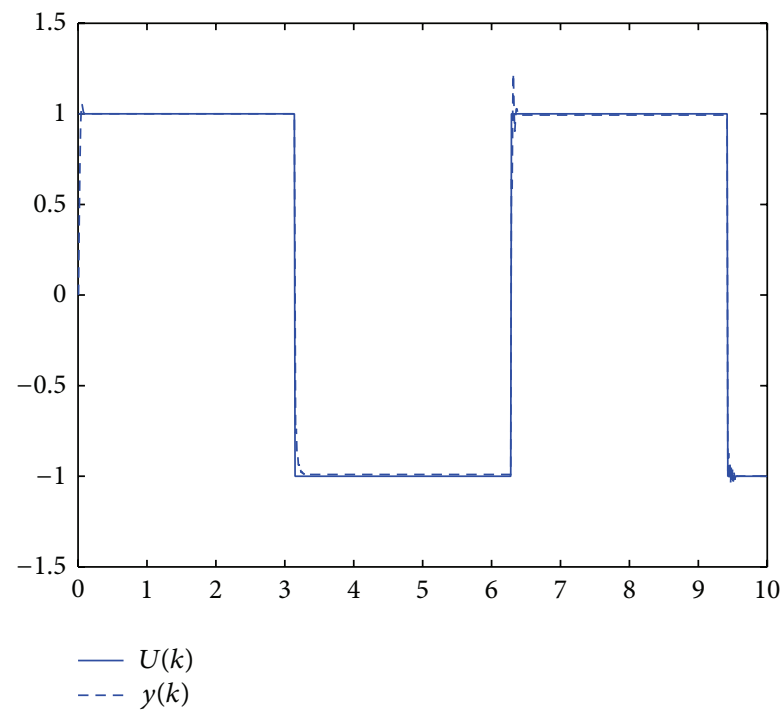

FIGURE 6: The response curve of square wave signal.

signal as the input signal, the tracking curve diagram of the system is as shown in Figures 7 and 8.

Model 4. Consider the following time-varying system: $y(k)=$ $\alpha_{0}\left(k-d_{0}\right)+\alpha_{1}\left(k-d_{1}\right) u(k-1)+\alpha_{2}\left(k-d_{2}\right) u^{2}(k-1)$, where $\alpha_{0}\left(k-d_{0}\right)=0.2 y(k-1) u(k-2)+0.5 u^{3}(k-1), d_{0}=2$; $\alpha_{1}\left(k-d_{1}\right)=-0.4 y(k-2), d_{1}=0 ; \alpha_{2}\left(k-d_{2}\right)=0.5 y(k-1)$, $d_{2}=0$. The nonlinear model of the plant is obtained: $y(k)=$ $0.2 y(k-1) u(k-2)-0.4 y(k-2) u(k-1)+0.5 y(k-1) u^{2}(k-$ $1)+0.5 u^{3}(k-1)$. After choosing sine and square wave signal as the input signal, the tracking curve diagram of the system is as shown in Figures 9 and 10.

It can be seen from the four simulation models above that U-model has a simple mathematical form; the identification method of adaptive neural network has high accuracy of identification, and it can be used to obtain precise parameters

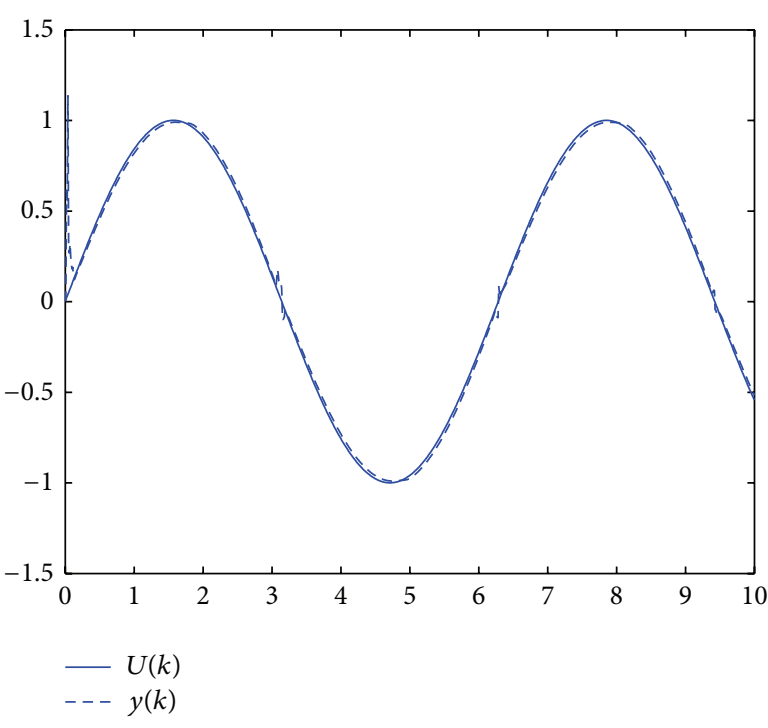

FIGURE 7: The response curve of sine signal.

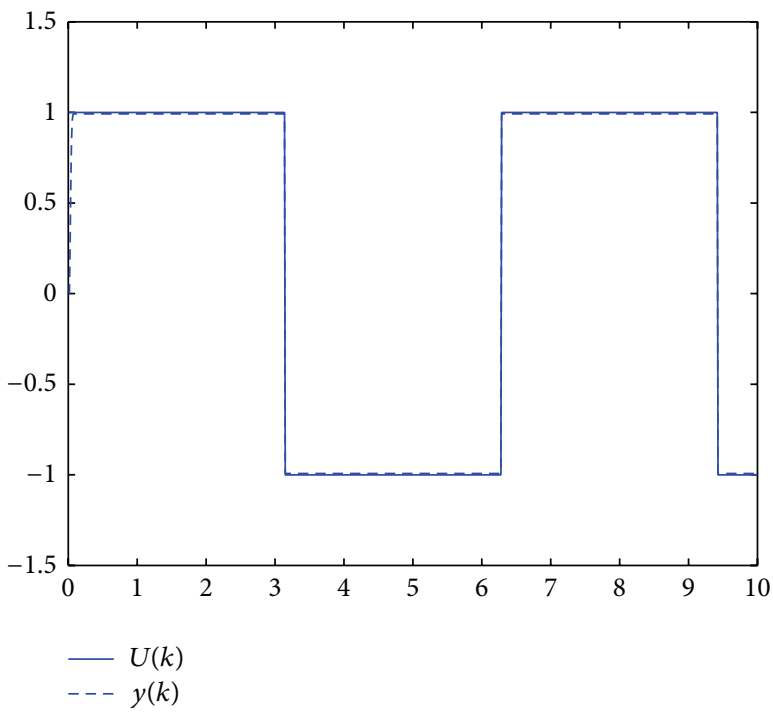

FIGURE 8: The response curve of square wave signal.

for the design of adaptive controller, so the adaptive controller designed has high control precision for nonlinear timevarying delay systems. The output of the system can track the input accurately, achieving the control of high precision for nonlinear time-varying delay systems.

\section{Conclusions}

The method of the nonlinear adaptive neural network control system based on U-model is presented in this paper; it has solved a class of nonlinear time-varying delay problems about the identification and the design of control system. U-model describes the nonlinear plant by the time-varying delay polynomial, which constructs a general and high accuracy nonlinear model and provides a precise mathematical model for the design of nonlinear time-varying delay systems. 


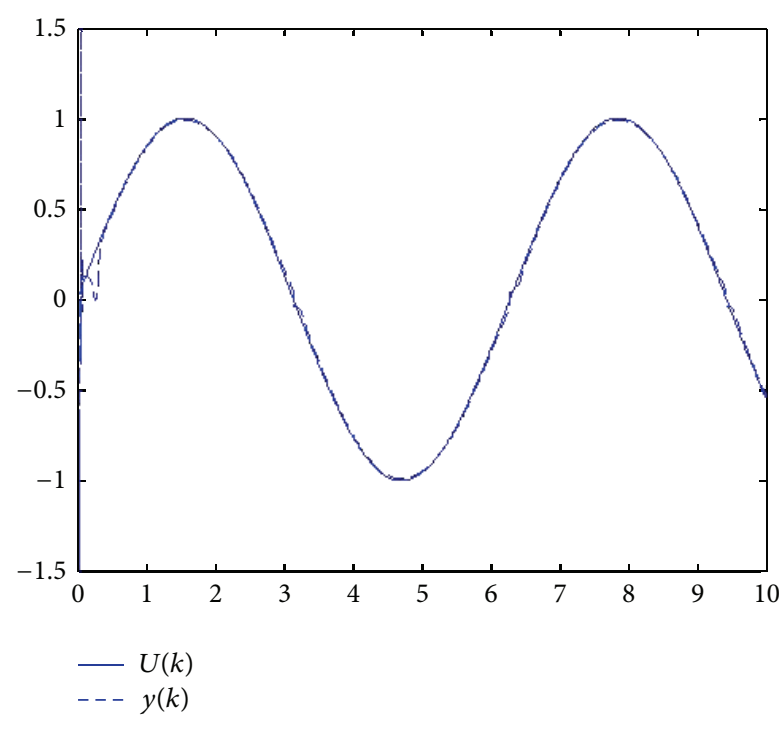

FIGURE 9: The response curve of sine signal.

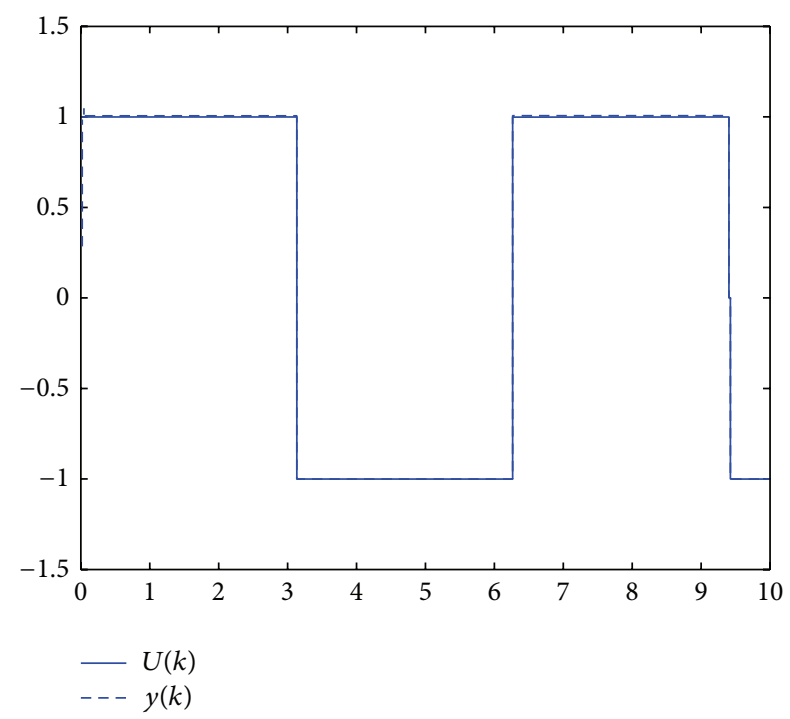

FIGURE 10: The response curve of square wave signal.

On this basis, the algorithm of adaptive neural network identification based on U-model is proposed, which uses a general time-varying delay nonlinear model to identify the nonlinear plant online and provides a theoretical basis for the establishment of unknown nonlinear time-varying delay plant model and the study of the identification methods of nonlinear time-varying delay systems. The adaptive neural network algorithm presented in this paper provides a new research direction for online identification of nonlinear time-varying delay system which used the Newton-Raphson formula to design the controller of nonlinear time-varying delay systems based on the identification of nonlinear Umodel. Performance analysis of nonlinear time-varying delay control systems is given, and the stability of control system is proved by analysis of the real root.

\section{Conflict of Interests}

The authors declare that there is no conflict of interests regarding the publication of this paper.

\section{Acknowledgment}

This work was supported in part by the project of Heilongjiang Province Education Department Research Project under Grant 12511604.

\section{References}

[1] L. Wu, Z. Feng, and W. X. Zheng, "Exponential stability analysis for delayed neural networks with switching parameters: average dwell time approach," IEEE Transactions on Neural Networks, vol. 21, no. 9, pp. 1396-1407, 2010.

[2] L. Wu, X. Su, P. Shi, and J. Qiu, "A new approach to stability analysis and stabilization of discrete-time T-S fuzzy timevarying delay systems," IEEE Transactions on Systems, Man, and Cybernetics B: Cybernetics, vol. 41, no. 1, pp. 273-286, 2011.

[3] Y. He, M. Wu, J. H. She, and G. P. Liu, "Parameter-dependent Lyapunov functional for stability of time-delay systems with polytopic-type uncertainties," IEEE Transactions on Automatic Control, vol. 49, no. 5, pp. 828-832, 2004.

[4] C. Lin, Q. Wang, and T. H. Lee, "Stability and stabilization of a class of fuzzy time-delay descriptor systems," IEEE Transactions on Fuzzy Systems, vol. 14, no. 4, pp. 542-551, 2006.

[5] B. Xu and Y. Liu, "Delay-dependent/delay-independent stability of linear systems with multiple time-varying delays," IEEE Transactions on Automatic Control, vol. 48, no. 4, pp. 697-701, 2003.

[6] D. Yue, J. Fang, and S. Won, "Delay-dependent robust stability of stochastic uncertain systems with time delay and Markovian jump parameters," Circuits, Systems, and Signal Processing, vol. 22, no. 4, pp. 351-365, 2003.

[7] Y. He, G. Liu, and D. Rees, "New delay-dependent stability criteria for neural networks with time-varying delay," IEEE Transactions on Neural Networks, vol. 18, no. 1, pp. 1045-9227, 2007.

[8] C. Onat, "A new concept on PI design for time delay systems: weighted geometrical center," International Journal of Innovative Computing, Information and Control, vol. 9, no. 4, pp. 15391556, 2013.

[9] L. Wu, X. Su, and P. Shi, "Output feedback control of Markovian jump repeated scalar nonlinear systems," IEEE Transactions on Automatic Control, vol. 59, no. 1, pp. 199-204, 2014.

[10] R. Yang, P. Shi, G. Liu, and H. Gao, "Network-based feedback control for systems with mixed delays based on quantization and dropout compensation," Automatica, vol. 47, no. 12, pp. 2805-2809, 2011.

[11] L. Wu, X. Su, P. Shi, and J. Qiu, "Model approximation for discrete-time state-delay systems in the TS fuzzy framework," IEEE Transactions on Fuzzy Systems, vol. 19, no. 2, pp. 366-378, 2011.

[12] C. Han, L. Wu, H. K. Lam, and Q. Zeng, "Non-fragile control with guaranteed cost of T-S fuzzy singular systems based on parallel distributed compensation," IEEE Transactions on Fuzzy Systems, 2013.

[13] R. Yang, G. Liu, P. Shi, C. Thomas, and M. V. Basin, "Predictive output feedback control for networked control systems," IEEE 
Transactions on Industrial Electronics, vol. 61, no. 1, pp. 512-520, 2014.

[14] P. Shi, E. K. Boukas, and R. K. Agarwal, "Control of Markovian jump discrete-time systems with norm bounded uncertainty and unknown delay," IEEE Transactions on Automatic Control, vol. 44, no. 11, pp. 2139-2144, 1999.

[15] L. Xie, E. Fridman, and U. Shaked, "Robust $H_{\infty}$ control of distributed delay systems with application to combustion control," IEEE Transactions on Automatic Control, vol. 46, no. 12, pp. 1930-1935, 2001.

[16] G. F. Zou and Z. O. Wang, "Identification of nonlinear time varying systems based on recurrent neural networks," Control and Decision, vol. 17, no. 5, pp. 517-521, 2002.

[17] G. Pillonetto, "Identification of time-varying systems in reproducing kernel Hilbert spaces," IEEE Transactions on Automatic Control, vol. 53, no. 9, pp. 2202-2209, 2008.

[18] T. Hsiao, "Identification of time-varying autoregressive systems using maximum a posteriori estimation," IEEE Transactions on Signal Processing, vol. 56, no. 8, pp. 3497-3509, 2008.

[19] H. Liu, J.-A. Lu, J. Lü, and D. J. Hill, "Structure identification of uncertain general complex dynamical networks with time delay," Automatica, vol. 45, no. 8, pp. 1799-1807, 2009.

[20] Q. M. Zhu and L. Z. Guo, "A pole placement controller for nonlinear dynamic plants," Proceedings of the Institution of Mechanical Engineers I: Journal of Systems and Control Engineering, vol. 216, no. 6, pp. 467-476, 2002.

[21] M. Shafiq and N. R. Butt, "Real-time adaptive tracking of DC motor speed using U-model based IMC," Automatic Control and Computer Sciences, vol. 41, no. 1, pp. 31-38, 2007.

[22] T. Khan and M. Shafiq, "A novel internal model control scheme for adaptive tracking of nonlinear dynamic plants," in Proceeding of the 1st IEEE Conference on Industrial Electronics and Applications (ICIEA '06), pp. 1-6, Singapore, May 2006.

[23] X. L. Wu, L. Liu, Q. M. Zhu, W. Du, B. Wang, and J. Zhang, "U-model-based adaptive control for a class of stochastic nonlinear dynamic plants with unknown parameters," International Journal of Modelling, Identification and Control, vol. 13, no. 3, pp. 135-143, 2011.

[24] F. Xu, Q. Zhu, D. Zhao, and S. Li, "U-model based design methods for nonlinear control systems a survey of the development in the 1st decade," Control and Decision, vol. 28, no. 7, pp. 961977, 2013.

[25] Q. M. Zhu, K. Warwick, and J. L. Douce, "Adaptive general predictive controller for nonlinear systems," IEE Proceedings D: Control Theory and Applications, vol. 138, no. 1, pp. 33-40, 1991. 


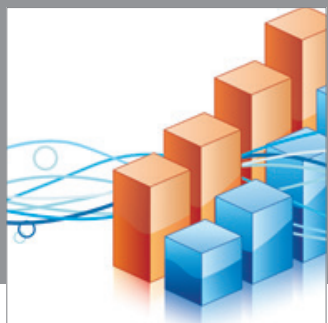

Advances in

Operations Research

mansans

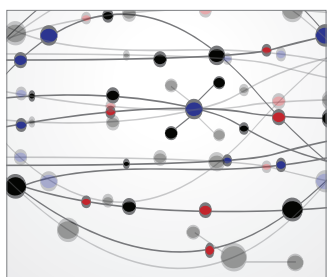

The Scientific World Journal
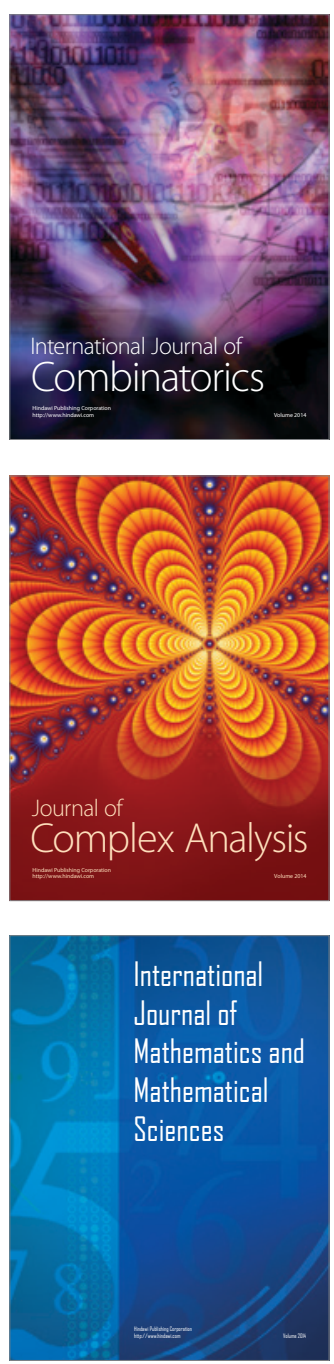
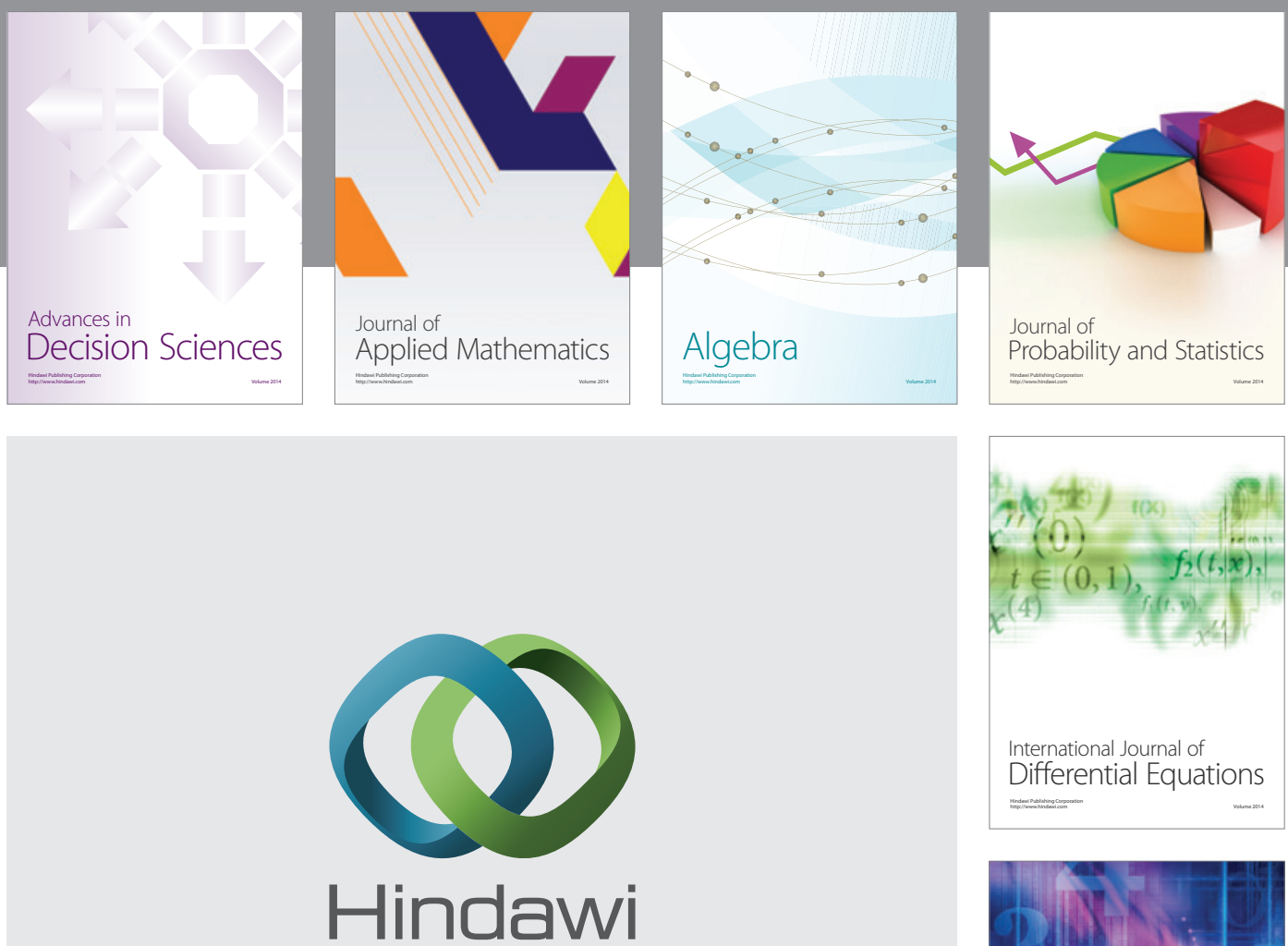

Submit your manuscripts at http://www.hindawi.com
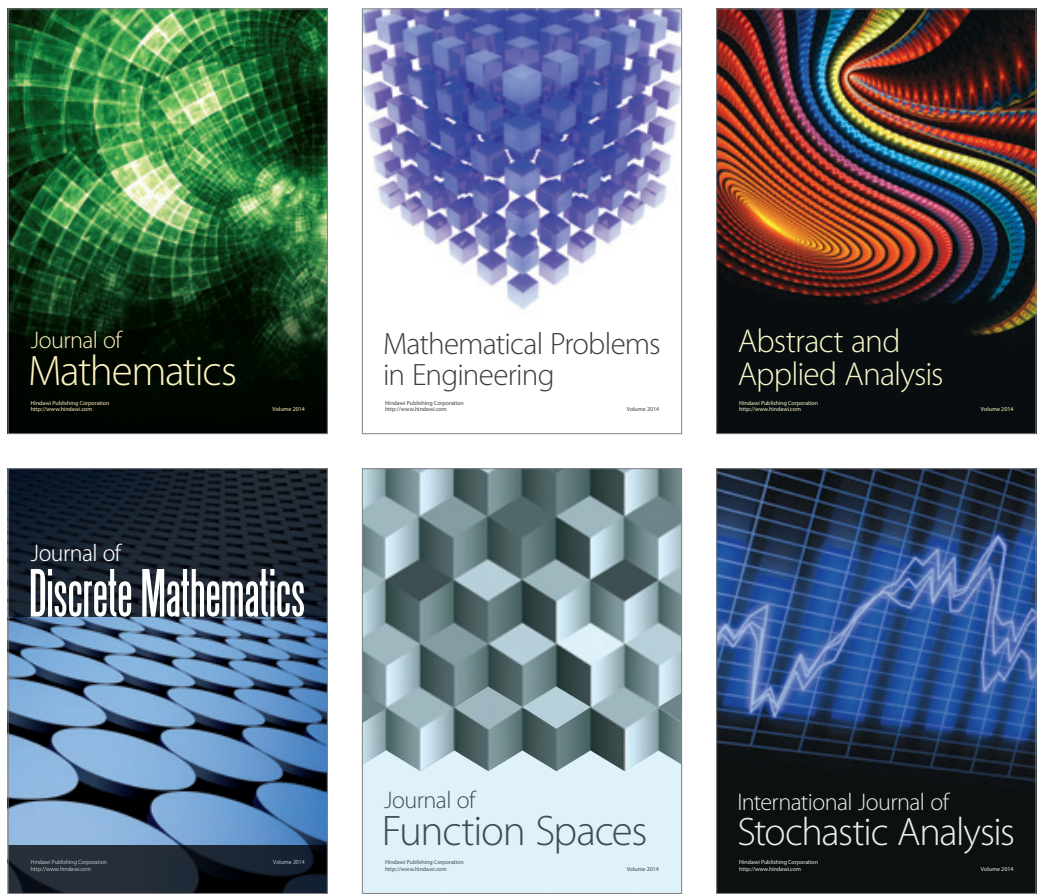

Journal of

Function Spaces

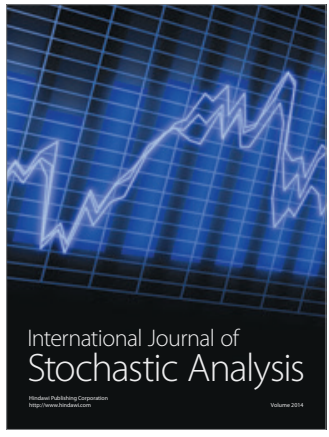

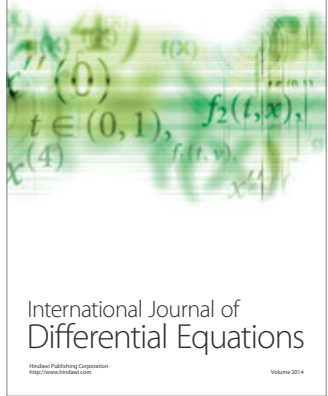
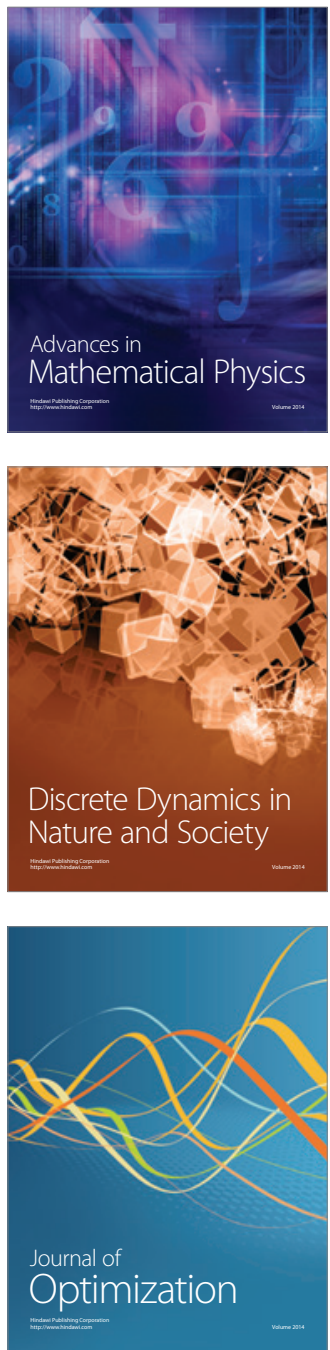